\title{
6. EMPIRICAL STUDY ON EFFECTIVENESS OF HDTV IN DISTANCE EDUCATION
}

\author{
Hiroshi Kato, Mitsushige Iwamoto, \\ and Hideki Asahi \\ C\&C Media Research Laboratories, \\ NEC Corporation \\ 4-1-1, Miyazaki, Miyamae-ku, \\ Kawasaki-shi, 216-8555, Japan \\ E-mail:kato@ccm.cl.nec.co.jp
} Phone: +8-427-44-7527, Fax: +8-44-856-2235,

\begin{abstract}
A distance learning experiment was conducted to examine differences in learning performance and to obtain subjective evaluations of three types of learning methods: face-to-face (LIVE), distance learning using high-definition television (HDTV), and distance learning using normal TV (NTSC). Five sites were used for HDTV and six sites for NTSC, where the same lecture was watched at the same time. There were 74 voluntary participants for the LIVE lecture, 144 for HDTV, and 139 for NTSC. Based on pretest, posttest, and questionnaire results, the authors found that, as far as the learners' performance level is concerned, the LIVE method is more effective than the others, while there was no significant difference between HDTV and NTSC. It was also found that HDTV is better at showing the atmosphere of the lecture, and motivates learners more than NTSC. Third, HDTV still has difficulty in showing a highresolution computer screen, though it is perceived better than NTSC. As for other points, HDTV's image quality is satisfactory to learners as a whole.
\end{abstract}

\section{Keywords \\ Distance Learning, Communications, Multimedia, Satellites, Video Conferencing}

The original version of this chapter was revised: The copyright line was incorrect. This has been corrected. The Erratum to this chapter is available at DOI: 10.1007/978-0-387-35393-7_22 


\section{Introduction}

Recent advancements in communication technology have made distance learning systems more economical and more efficient. Since distance learning systems allow learners to attend lectures by eminent lecturers with less transportation costs, they have become popular with universities (e.g. Shimizu et al. 1986, Tanaka 1993, Yamasawa et al. 1991) and companies (Shiratori 1988).

In these systems, TV-based teleconference systems are utilized. The image quality from current TV standards such as PAL and NTSC, however, is not clear enough to show characters on a white board or on a computer screen legibly. Therefore, high-definition television (HDTV), which has approximately twice as many scanning lines (1125 lines) as compared to NTSC (525 lines), is expected to be a core technology for the next generation of distance learning systems.

Shimizu and Shiroma (Shimizu et al. 1989, 1990, 1995) investigated distance learning using HDTV, and found that HDTV was evaluated better than NTSC on two points: 'atmosphere of the lecture' and 'quality of the image.' In these experiments, however, learner's performance was not examined. This is probably because the content of the learning was different for NTSC and HDTV, so learner's performance levels could not be directly compared.

Although it was not a case of distance education, Iimori et al. (limori et al. 1991, 1993) conducted experiments using video programs for school education to examine the differences in cognitive performance and impressions between HDTV and NTSC. They showed subjects a short (approx. 15 minutes) video program produced for broadcasting, and performed recognition and memory tests concerning the contents of the program. In one of their experiments using a drama program, students watching HDTV achieved higher performance in understanding and memory tests, but in another experiment using a verbally explanatory program, no significant difference was found in understanding and memory test (Iimori et al. 1993). However, we should be careful in assuming that these results would be the same in the case of distance learning, because distance education includes some other factors such as difference in instructional method, the possibility of interaction with a lecturer, and the sense of simultaneous presence with a lecturer and other learners at other sites.

In our experiment, we used a three-hour lecture given live and transmitted at the same time through an NTSC-based distance learning system and an HDTV-based distance learning system. We conducted pre- and posttests concerning the content of the lecture and a questionnaire for learners' subjective evaluations of the three learning methods in order to analyze the differences in learners' learning performance levels and motivation, impressions, etc., respectively. 


\section{Experiment}

\subsection{Distance learning system}

The lecture was held at a central lecture room in Tokyo. HDTV signals of the scenes were transmitted from an up-link van parked outside the room to five remote sites via the JCSAT-1 communication satellite (Figure 1). The video and audio signals were transmitted by MUSE and B-mode stereo, respectively. On the other hand, common TV (NTSC) system was based on NESPAC; NESPAC is a commercial two-way distance learning system employing a communication satellite (Shiratori 1988). The NTSC signals were transmitted to the Tokyo NESPAC site via a microwave link, and to six remote sites via JCSAT-1. The source signal for NTSC was generated from an HDTV signal with an HDTV-NTSC converter, so that the lecture was the same for all remote sites.

The equipment used in the experiment included video cameras, a document camera, a monitor screen $(1280 \times 1024)$ of an engineering workstation (EWS) downconverted to HDTV, and an HDTV (Uni-hi) VCR.

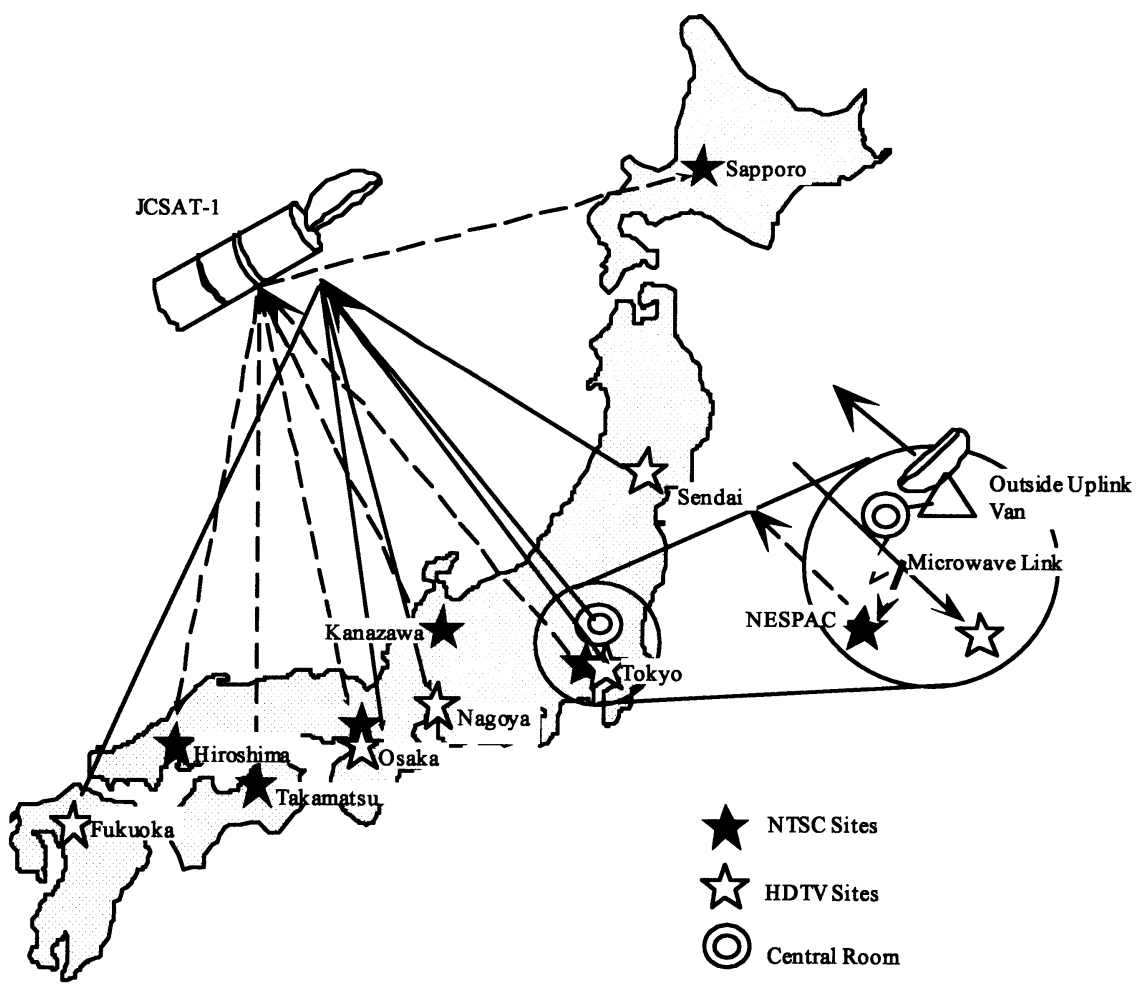

Figure 1 Distance learning system used in the experiment. 


\section{$2.2 \quad$ Learning conditions}

The major factor in the experiment was the types of learning methods. Based on the learning methods, conditions were divided into three categories: face-to-face learning, distance learning using HDTV, and distance learning using NTSC. We refer to these conditions respectively as 'LIVE', 'HDTV' and 'NTSC'. Table 1 shows the condition, number of subjects, size of the monitor screen, and room configuration at each site.

Table 1 Overview of the experiment design

\begin{tabular}{|c|c|c|c|c|c|}
\hline \multirow{2}{*}{$\begin{array}{l}\text { Condition } \\
\text { LIVE }\end{array}$} & \multirow{2}{*}{$\begin{array}{c}\begin{array}{c}\text { Location } \\
\text { of sites } \\
\text { (city) }\end{array} \\
\text { Tokyo }\end{array}$} & \multirow{2}{*}{$\begin{array}{r}\begin{array}{c}\text { Screen } \\
\text { size } \\
\text { (inch) }\end{array} \\
110\end{array}$} & \multirow{2}{*}{$\begin{array}{c}\text { Room configuration } \\
\text { (The \# of seats per desk } * \text { the \# of } \\
\frac{\text { desks per column for the first column }}{+(\ldots) \text { for the } 2 n d+\ldots} \\
\left(3^{*} 9\right)+\left(10^{*} 9\right)+\left(3^{*} 9\right)\end{array}$} & \multicolumn{2}{|c|}{$\begin{array}{l}\text { Number of } \\
\text { subjects } \\
\text { (\# of missing } \\
\text { values in } \\
\text { pre/post-test) }\end{array}$} \\
\hline & & & & $74(1)$ & $74(1)$ \\
\hline \multirow{5}{*}{ HDTV } & Sendai & 54 & $(3 * 4)^{*} 3$ & $19(0)$ & \multirow{5}{*}{$144(5)$} \\
\hline & Tokyo 1 & 61 & $\left(3^{*} 3\right)+\left(3^{*} 6\right)^{*} 3$ & $62(0)$ & \\
\hline & Nagoya & 54 & $\left(3^{*} 5\right)^{*} 3$ & $15(2)$ & \\
\hline & Osaka 1 & 54 & $(3 * 4)^{*} 3$ & $31(2)$ & \\
\hline & Fukuoka & 54 & $(3 * 4)^{*} 3$ & $17(1)$ & \\
\hline \multirow{6}{*}{ NTSC } & Sapporo & 70 & $\left(3^{*} 4\right)^{*} 2$ & $19(0)$ & \multirow{6}{*}{ 139(6) } \\
\hline & Tokyo 2 & 70 & $\left(3^{*} 5\right)^{* 3}$ & $9(1)$ & \\
\hline & Kanazawa & 70 & $\left(3^{*} 3\right)+\left(3^{*} 4\right)^{* 2}$ & $31(2)$ & \\
\hline & Osaka 2 & 46 & $\left(3^{*} 6\right)^{*} 3$ & $41(0)$ & \\
\hline & Hiroshima & 70 & $(3 * 5)^{* 3}$ & $31(3)$ & \\
\hline & Takamatsu & 70 & $(3 * 4)^{* 2}$ & $8(0)$ & \\
\hline
\end{tabular}

All of the remote sites were lecture rooms for the NESPAC distance education system. Therefore, illumination, desk arrangement, screen configuration (every room had two screens), etc., were carefully designed so that every seat had a good view of the screens. The distance from the screens to the first row of seats was 2 through $3 \mathrm{~m}$.

However, as shown in Table 1, only Osaka2 (NTSC) had smaller screens in relative to its room size, so let us examine if it affected the results. $>$ From the results of the questionnaire items on screen view, as determined by the items Q4 through Q12 (Figure 4), there was no significant difference between NTSC sites. The results of analysis of variance (ANOVA) are shown in Table 2. Of these items, Q8 and Q11 were nearly significant, but Osaka2 was the fourth best of all six NTSC sites in both items, so the screen size in this case was determined not to have mattered. Consequently, we decided that Osaka2 did not have to be eliminated from the data. 
Table 2 Results of ANOVA for NTSC sites

\begin{tabular}{llllllllll}
\hline & $Q 4$ & $Q 5$ & $Q 6$ & $Q 7$ & $Q 8$ & $Q 9$ & $Q 10$ & $Q 11$ & $Q 12$ \\
\hline df & $5 /$ & $5 /$ & $5 /$ & $5 /$ & $5 /$ & $5 /$ & $5 /$ & $5 /$ & $5 /$ \\
& 131 & 131 & 132 & 133 & 133 & 132 & 133 & 132 & 132 \\
F & 1.49 & 1.61 & 1.84 & 1.75 & 2.06 & .733 & 1.39 & 2.27 & 0.78 \\
p & .200 & .162 & .108 & .129 & .074 & .600 & .232 & .051 & .566 \\
\hline
\end{tabular}

HDTV monitors were installed 0.5 through $1 \mathrm{~m}$ in front of the front seats, so the screen was nearer to the learners than the NTSC screens, so as to compensate for the smaller screen of HDTV monitors. In the HDTV condition, only Tokyol had two HDTV monitors. This is why the width of the room was a desk-width longer than the other rooms. In the same way, the screen size at Tokyol was seven inches bigger because the depth of the room was one or two desk-depths longer. The viewing conditions of HDTV remote sites were thus controlled.

\subsection{Subjects}

All of the subjects were employees of NEC or affiliated companies aged from 20 to 35. All of them were voluntary applicants for the following learning course specially provided free of charge, which was announced in an ordinary manner as employee education program. In Tokyo and Osaka, both distance learning systems were tested in the same buildings, so subjects are distributed randomly in two sites of Tokyo and Osaka each before the actual experiment. The numbers of subjects at each site are shown in Table 1.

\section{$2.4 \quad$ Lecture}

The theme of the lecture was 'Introduction to GUI (Graphical User Interface).' This theme was selected because it requires high-resolution image $(1280 \times 1024)$ of computer operations to be shown on a monitor screen.

The lecture was performed for three hours, including short breaks, in the afternoon on February 22nd, 1993. A brief question-and-answer period was taken at the end of the lecture. The questioner's image from a remote site was transmitted to the central lecture room in NTSC, and then distributed to the other sites.

\subsection{Apparatus}

(1) Pretest and posttest. Pretests and posttests were designed to investigate the performance of the learners under each condition. The pretest consisted of 13 items directly related to the content of the lecture. The posttest included all items of the pretest and three questions on learners' prior knowledge of the theme. Three minutes were given to take the tests.

(2) Questionnaire. A questionnaire was designed for learner's subjective evaluation of various aspects of the experiments. It consisted of 32 items for LIVE, and 36 items for NTSC and HDTV. The items included questions about installation 
of the monitor screen, legibility, audibility, impressions of the lecture, quality of the lecture, and familiarity with the lecturer. The items are listed in Figure 4.

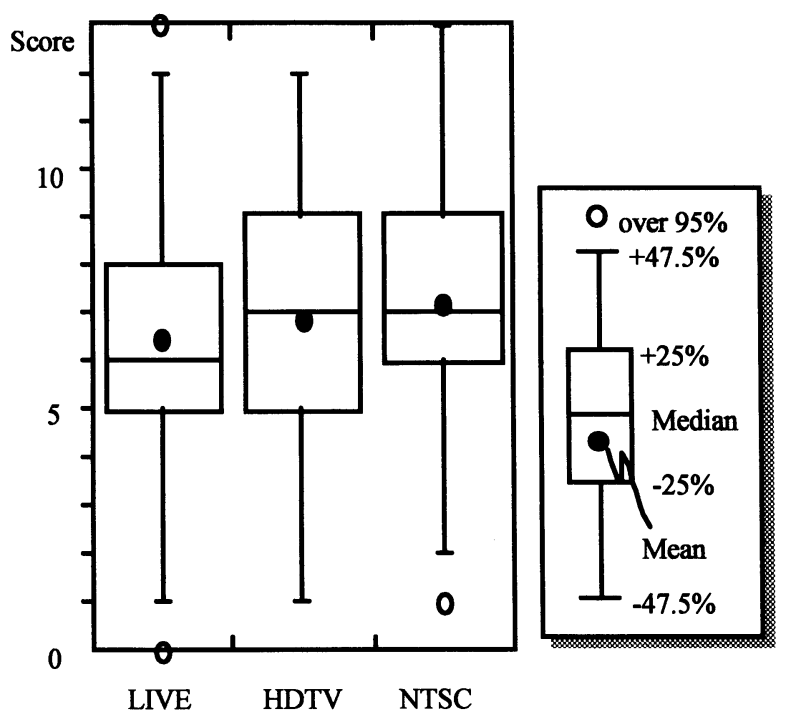

Figure 2 Mean of pretest scores of each site.

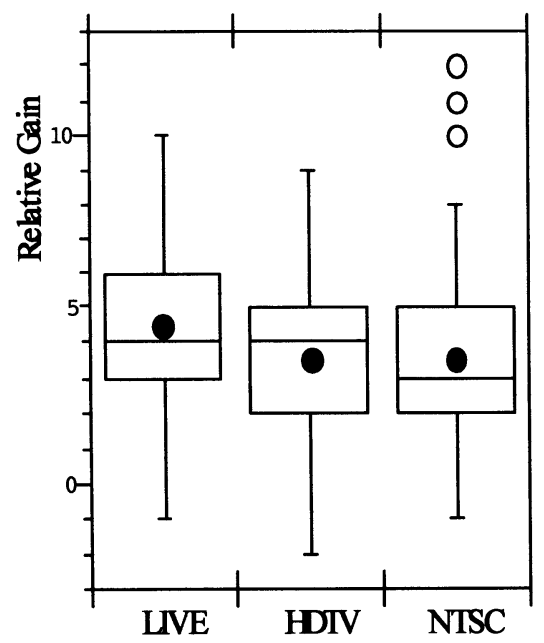

Figure 3 Distribution of relative gains of each condition. 


\section{Results and discussion}

\subsection{Equality of learners regarding subject matter}

The learners' equality among conditions in terms of their prior knowledge on the lecture subject matter is discussed. Figure 2 shows the distribution of pretest scores for each condition. Through an ANOVA, the hypothesis that pretest score means for each condition are equal cannot be rejected $(\mathrm{F}(2,343)=2.27 ; \mathrm{p}=0.11)$. Thus, there is no evidence of a difference in the learners' readiness between conditions.

\subsection{Analysis of performance test}

Table 3 AIC of Null model (0), Method model (1), and Site model (2)

\begin{tabular}{|c|c|c|c|c|c|c|c|c|c|}
\hline \multirow{2}{*}{ Model } & \multirow{2}{*}{$\begin{array}{l}\text { Number of } \\
\text { parameters }\end{array}$} & \multicolumn{7}{|c|}{ Maximum likelihood estimators } & \multirow{2}{*}{$A I C$} \\
\hline & & $\mu$ & $\sigma^{2}$ & & & & & & \\
\hline (0) & 2 & 3.72 & 5.11 & & & & & & 1545.9 \\
\hline (1) & 4 & 3.84 & 4.96 & .6 & & -.3 & & -.318 & 1539.7 \\
\hline \multirow{2}{*}{ (2) } & \multirow{2}{*}{13} & \multirow{2}{*}{4.59} & \multirow{2}{*}{4.94} & & -.32 & .03 & .03 & $-.24 \quad .16$ & \multirow{2}{*}{1555.9} \\
\hline & & & & -.21 & -.09 & .21 & .02 & $\begin{array}{ll}-.37 & -.09\end{array}$ & \\
\hline
\end{tabular}

To examine an individual learner's performance, we calculated relative gains, which is defined as the posttest score minus the pretest score. Figure 3 shows the distribution of relative gains in each condition. There was a significant difference among the means in relative gains $(F(2,342)=5.12 ; p=0.006)$. However, it should be discussed which factor - learning method or site - is more likely to account for the data beforehand. Consequently, Akaike Information Criterion (AIC) (Akaike 1974) values of the following three models are compared as shown in Table 3, where AIC represents how well a stochastic model fits data, taking the number of parameters used in the model into account.

Null model (0): All the data are samples from $\mathrm{N}\left(\mu_{0}, \sigma_{0}^{2}\right)$.

Method model (1): For each condition ( $\mathrm{i}=1 \ldots 3)$, the data are samples from $\mathrm{N}\left(\mu_{0}+\alpha_{\mathrm{i}}\right.$, $\left.\sigma_{0}^{2}\right)$, where $\Sigma \alpha_{\mathrm{i}}=0$.

Site model (2): For each site $(\mathrm{j}=1 \ldots 12)$, the data are samples from $\mathrm{N}\left(\mu_{0}+\alpha_{\mathrm{j}}, \sigma_{0}{ }^{2}\right)$, where $\Sigma \alpha_{j}=0$.

An AIC value of the ANOVA model is given by the following formula (Suzuki 1995), where $\sigma^{2}, n$, and $c$ is maximum likelihood estimator of the variance, the number of samples, and the number of parameters used in the model, respectively.

As shown in Table 3, the models have the following relationship based on their AIC values. 
Site model (1) < Null model (0) < Method model (2)

As a result, it is confirmed that the learning method factor influenced the learner's performance, while the site factor was not significant.

From the result, we concluded that the learning method factor was significant. Therefore, it is essential to identify the differences in methods. Table 4 shows the results of multiple comparisons and the mean of relative gains for each condition. As shown, LIVE is more effective than the others, while there is very little difference between HDTV and NTSC.

Table 4 Multiple comparison tests between conditions

\begin{tabular}{|c|c|c|c|}
\hline & LIVE & HDTV & Mean of relative gain \\
\hline LIVE & & & 4.466 \\
\hline HDTV & $.029 *$ & & 3.525 \\
\hline NTSC & $.028^{*}$ & 1.000 & 3.519 \\
\hline
\end{tabular}

In the precedent study by limori et al. (Iimori et al. 1993), as mentioned earlier, subject groups who watched a video program depending much for explanation did not show any differences in memory and understanding between HDTV and NTSC. From our result that the difference in quality of visual information was not influential, the lecture in this experiment was considered to depend basically for lecturer's verbal explanation as well, even though many visual aids, such as document camera and computer screen, were used. This result implies that the lecture-like style of instructional method used in the experiment, although it is generally used in distance education, cannot take full advantage of high definition of HDTV in terms of the learning performance.

\subsection{Analysis of subjective evaluation}

First of all, we tried to categorize the items according to the tendencies of their response patterns. By applying factor analysis to the HDTV and NTSC data, we extracted eleven factors whose eigenvalues were larger than 1.0. Items in the questionnaire were categorized according to the factor loading after vari-max rotation. Figure 4 shows the items grouped by horizontal lines according to the factors and the means of responses for each condition. 


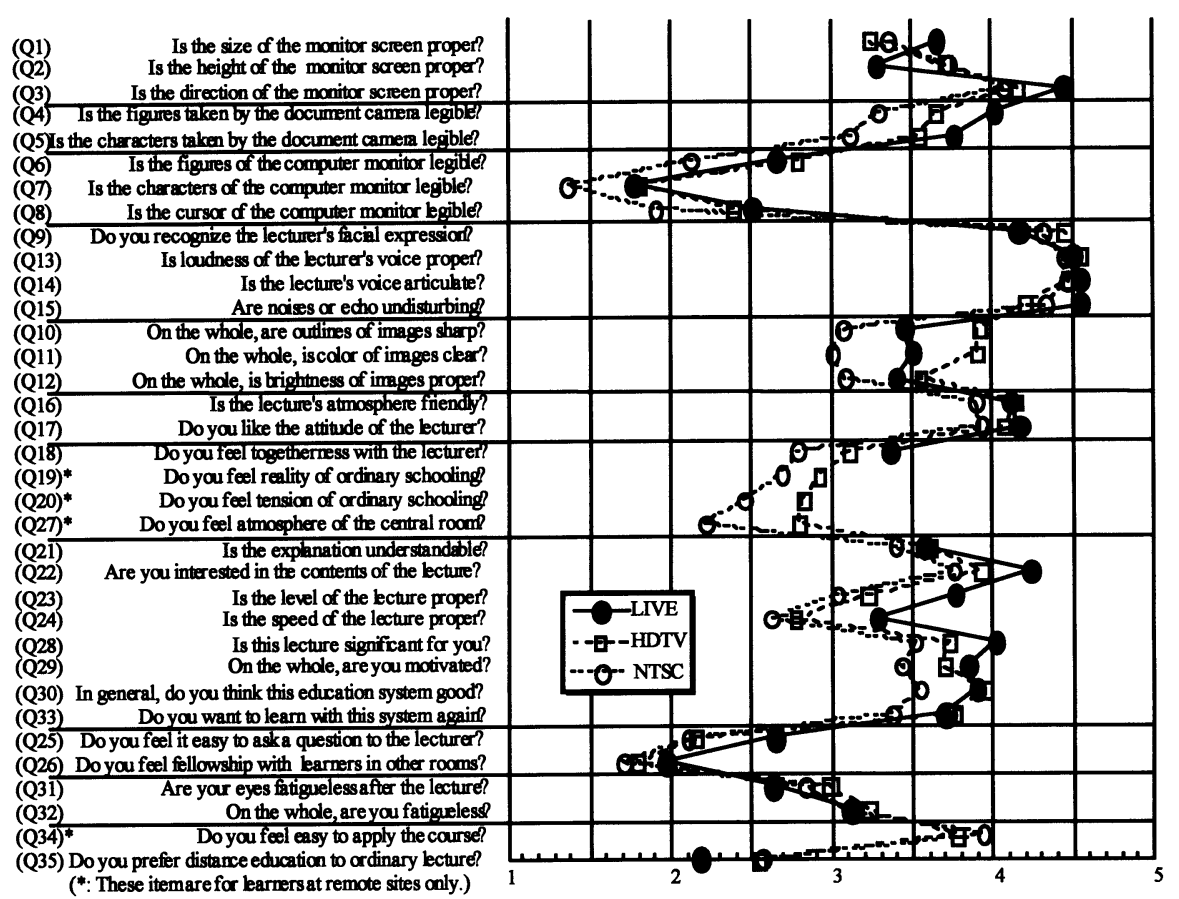

Figure 4 Results of the questionnaire. (The original questions were in Japanese.) In general, as shown in Figure 4, there was no problem with audio [Q13, Q14,

Q15], and video images were sufficient for seeing the lecturer's face [Q9] and fair for reading documents [Q4, Q5]. However, the legibility of the computer monitor screen was poor in all conditions [Q6, Q7, Q8]. Overall, there was a favorable impression of the lecture [Q21, Q22, Q23, Q28, Q29] except for its speed [Q24]. The lecturer was friendly [Q16, Q17], but putting a question to the lecturer was not very easy [Q25]. The learners did not feel any belongingness with other learners [Q26], and learners in remote sites did not feel as if they were actually at the central room [Q18, Q19, Q20, Q27]. The learners said that this educational system is good [Q30], and that they may learn by this system again [Q33], but prefer a live lecture to distance learning [Q35].

The items that showed a significant difference between HDTV and NTSC are as follows.

$\mathrm{Q} 4 *, \mathrm{Q} 5 *, \mathrm{Q} 6 * *, \mathrm{Q} 7 * *, \mathrm{Q} 8^{* *}, \mathrm{Q} 10^{* *}, \mathrm{Q} 11^{* *}, \mathrm{Q} 12^{* *}, \mathrm{Q} 16^{*}, \mathrm{Q} 20^{* *}, \mathrm{Q} 27^{* *}$, Q29*, Q30**, Q33** $\quad(* *: \mathrm{p}<0.01, *: \mathrm{p}<0.05)$

This means that HDTV is significantly better than NTSC in viewing documents [Q4*, Q5*] and monitor screens $\left[\mathrm{Q}^{* *}, \mathrm{Q}^{* *}, \mathrm{Q} 8^{* *}\right]$, although the image of the computer screen is not entirely satisfactory [Q6, Q7, Q8]. On the whole, HDTV's superiority in the quality of the images was appreciated [Q10**, Q11**, Q12**]. 
Moreover, the atmosphere of the lecture was friendlier [Q16*] and more realistic [Q20**, Q27**] in HDTV. HDTV learners were more motivated and had a more favorable impression of the distance learning system [Q29*, Q30**, Q33**].

As for the sense of simultaneous presence with the lecturer and other learners at other sites - which is never available by an off-line video system, a certain improvement was shown in HDTV when compared with NTSC [Q20**, Q27**], although it was not sufficient yet [Q18, Q19, Q20, Q27]. This result implies that the distance education needs to take full advantage of interactivity more than conventional lecture, so that the lecturer and learners can share the sense that they are taking part in the same lecture in real time.

Compared with Shimizu and Shiroma's research (Shimizu et al. 1990), HDTV's superiority in terms of the 'quality of the image' and 'atmosphere of the lecture', which roughly correspond to the items $\left[\mathrm{Q} 4 *, \mathrm{Q} 5^{*}, \mathrm{Q} 6^{* *}, \mathrm{Q} 7 * *, \mathrm{Q} 8 * *, \mathrm{Q} 10^{* *}\right.$, $\mathrm{Q} 11^{* *}, \mathrm{Q} 12^{* *}$ ] and [Q16*, Q20**, Q27**] respectively, was observed in our investigation as well. In addition to their findings, we found that the learners using HDTV were more motivated [Q29*, Q30**, Q33**] than NTSC. This is unlikely to be a Hawthorne effect (Roethlisberger et al. 1939), because instructions and activities of the learners were the same in all sites; the only difference between NTSC and HDTV was the difference between the monitors installed in the classrooms.

\section{Conclusions}

The same lecture was given live (face to face) and over distance learning systems, one using HDTV and the other using NTSC. A pretest, posttest, and questionnaire were conducted to examine the differences between them.

As far as learner's performance is concerned, the LIVE condition was significantly better than remote conditions, and very little difference was found between HDTV and NTSC.

From the learner's subjective evaluation, we found that even HDTV was not legible enough to display a high-resolution image of a computer screen. The size of characters on the monitor seems to be more dominant than the resolution of the screen, so using a device to enlarge a part of the monitor screen is desirable. On the whole, however, learners recognized that HDTV was apparently better in image quality than NTSC. Consequently, the reality of the lecture was increased, and, moreover, learners were more motivated with HDTV than with NTSC.

A problem in our experiment would be a period of learning. Considering actual use of distance education, three hours may not be long enough. At present, we have contrary views in terms of effects of a longer learning period; one is that learner's positive impressions toward HDTV take positive effects to their performance, and the other is that HDTV's superiority in impressions declines after a long experience. Thus, a longer version of the experiment remains as a part of future works. Another remaining work is about a style of education. This result implies that the conventional lecture cannot take full advantage of high definition of HDTV. It is plausible that there is a more suitable style for distance education than a lecture, so exploration for a better style is needed. 


\section{Acknowledgments}

This investigation was commissioned by the Hi-Vision Promotion Association Incorporated and performed in cooperation with Japan Hi-Vision Corporation and NTT. The authors would like to thank Dr. Masahiko Kurata and Mr. Hiroyuki Naito of NEC Corp. for their helpful advice, Mr. Hideyuki Suzuki of NEC Corp. for his help in processing the data, and Mr. Fujio Abe of NEC Corp. for his contribution to the lecture design.

\section{References}

Akaike, H (1974) A new look at the statistical model identification, IEEE Trans. Autom. Contr., AC-19 716-723

Iimori A et al.(1991) The use of hi-vision in education, The NHK Monthly Report on Broadcast Research, 41 10 14-27

Iimori A et al.(1993) Use of hi-vision TV in school broadcasts: from the results of a comparative survey with conventional TV, regarding effectiveness in education, The NHK Monthly Report on Broadcast Research, 436 28-39

Roethlisberger, F J and Dickson, W J (1939) Management and the worker, Cambridge, Mass.: Harvard University Press

Shimizu, Y and Maesako, T (1986) Evaluation of TV-lecture connecting two campuses by optical fiber cable, Trans. IEICE, J69-A 10 1181-1189

Shimizu, Y and Shiroma, S (1989) Evaluation of distance education between two campuses by HDTV, IEICE Technical Report, ET89-37 63-70

Shimizu, Y and Shiroma, S (1990) Evaluation of HDTV closed circuit system for distance education, J. ITE Japan, 44 12. 1717-1722

Shimizu Y et al (1995) Evaluation of a HDTV distance lecture transmitted by satellite, in Proc. the 11th Conf. on Educational Technology, E23 41-42

Shiratori T (1988) A two-way distance education system using advanced creative education: NESPAC, NEC Tech. Jour., 4110 139-146

Suzuki G (1995) Jouhouryou-kijun niyoru toukei kaiseki nyumon, Kodansha Scientific, Tokyo

Tanaka, M (1993) A trial on distance education and training through the PARTNERS network, IEICE Trans. Fundamental, E76-A 7 1195-1198

Yamasawa $\mathrm{K}$ et al (1991) Tele-education by using the cooperating system of the Shinshu University video and data network and INS-net64, IEICE Technical Report, ET91-78 2330

Hiroshi Kato joined NEC Corporation in 1983. From 1989 to 1990 , he was a visiting researcher at CSLI at Stanford University. He is currently a principal researcher at the C\&C Media Research Laboratories of NEC Corporation and visiting associate professor at Saitama University. His research interests are in the area of situated cognition and computer support for collaborative learning.

Mitsushige Iwamoto joined NEC Corporation in 1974. He has been a sales engineer and currently a system-engineering manager at the Kyushu System Center, C\&C Systems Market Development Division of NEC Corporation. 
Hideki Asahi joined NEC Corporation and has been engaged in educating operations of personal computers and its application programs. He is currently with C\&C System Education Division of NEC Corporation. 